\title{
Stage IA Intrahepatic Bile Duct Cancer AJCC v8
}

National Cancer Institute

\section{Source}

National Cancer Institute. Stage IA Intrahepatic Bile Duct Cancer A/CC v8. NCI Thesaurus. Code C134611.

Stage IA includes: T1a, N0, M0. T1a: Solitary tumor equal to or less than $5 \mathrm{~cm}$ without vascular invasion. N0: No regional lymph node metastasis. M0: No distant metastasis. (AJCC 8th ed.) 